\title{
L'analisi del codeswitching: uno strumento pedagogico nell'insegnamento dell'italiano lingua straniera
}

\author{
Rosa M . Torrens G uerrini
}

\begin{abstract}
W ith this article it is my aim to determine to what extent discourse analysis (from a conversational point of view) is applied to language teaching research (from the point of view of the communicative approach). The sphere of application of discourse analysis is invoked by the omnipresence of verbal exchange (conversation) in second language classes, which may be seen as paradigmatic situations of language contact. Conversation contains specific bifunctional strategies that operate simultaneously as learning/teaching and communicative strategies. Among these bifunctional strategies, code-switching represents one of the most important. I will present a specific type of code-switching, conversational code-switching, which will be illustrated through a side-by-side presentation of examples taken from bilingual and exolingual contexts. Finally, I will analyze the examples in order to draw some conclusions.
\end{abstract}

\section{Bifunzionalità dello scambio dialogico}

Per capire l'ambito di applicazione dell'analisi del discorso - secondo un approccio interazionista - nell'insegnamento delle lingue straniere risulta particolarmente interessante inquadrare un tale studio in una situazione di insegnamento di tipo comunicativo. Pur esistendo diverse versioni dell'approccio comunicativo, alcuni aspetti dello stesso risultano largamente accettati, come, per esempio, l'affrontare sin dai primi livelli le quattro abilità primarie (comprendere, parlare, leggere, scrivere). ${ }^{1} \mathrm{~A}$ partire da tali abilità si tracciano le cosiddette abilità integrate tra cui, probabilmente, quella di maggior spicco è il «dialogare». Tale abilità viene affrontata, in un certo senso, quotidianamente, tramite I'uso della L2 da parte dell'insegnante sin dal primo giorno di lezione, ${ }^{2}$ essendo nei primi tempi tollerato, in grado maggiore o minore, il passaggio alla L1 dei discenti e, naturalmente, le interferenze.

1. Vicinanza tipologica tra L1 eL2 permettendo.

2. In modo più diffuso, ovviamente, nei casi in cui la L1 ela L2 siano affini, come lo spagnolo o il catalano el'italiano. 
Risulta ovvio che i momenti in cui si stabilisce un dialogo non corrispondono perfettamente al momento di focalizzazione sull'insegnamento della lingua ma anche, e in parte, a momenti di semplice comunicazione tra l'insegnante e gli apprendenti.

Q uest'osservazione si desume da un assunto fondamentale: nelle nostre lezioni di lingue straniere la lingua veicolare, cioè quella che rende possibile lo scambio comunicativo tra l'insegnante e i discenti, coincide perfettamente con la lingua oggetto di apprendimento. Per questo motivo le strategie discorsive che si attivano durante l'interazione assumono inevitabilmente, in numerose occasioni, la doppia veste di strategie di comunicazione e di strategie di insegnamento/apprendimento.

\section{I fenomeni di cambiamento di lingua}

Le lezioni di lingue straniere rappresentano situazioni paradigmatiche di contatto di lingue, nelle quali si verificano con grande frequenza passaggi dalla lingua obiettivo alla lingua materna degli alunni, e viceversa, passaggi chiamati, nella letteratura anglofona, codeswitching, ovvero cambiamenti di lingua.

I cambiamenti di lingua (d'ora in poi CL) equivalgono a passaggi da una lingua all'altra i quali vanno differenziati dalle interferenze, che rappresentano invece l'intreccio, di vario tipo, tra due diversi codici linguistici. Le funzioni dei CL dipendono da parametri contestuali preesistenti e anche da quelli che essi modificano. Q ui presenteremo un unico tipo di cambiamento di lingua: I'alternanza, la quale, da un lato non dipende dalla modifica dei parametri contestuali (come per esempio, il tema, la costellazione dei partecipanti, ecc.); dall'altro rappresenta un cambiamento di lingua momentaneo - cioè non comporta il cambiamento della lingua base dell'interazione.

Esistono, in sostanza, due tipi di alternanza (N ussbaum, 1992), la cui frontiera viene tracciata in base al concetto di esolinguismo.

\section{II concetto di esolinguismo}

Secondo Alber - Py (1986), la situazione di insegnamento delle lingue straniere corrisponde alle cosiddette situazioni esolingui, vale a dire a situazioni nelle quali gli interlocutori si collocano in posizione asimmetrica a causa della loro diversa conoscenza del repertorio linguistico. È caratteristica di situazioni di disuguaglianza di vario genere che includono, oltre al rapporto parlante nativo/non nativo, il rapporto tra medico/paziente, adulto/bambino, ecc. T ale situazione, che preesiste alla stessa lezione di lingue straniere e attribuisce a ciascuno degli interlocutori uno status determinato, viene modificata in vari modi tramite i rapporti che si stabiliscono tra gli alunni e l'insegnante e che incidono sullo status stesso. Q uesto concetto verrà usato qui di seguito per spiegare i diversi tipi di cambiamento di lingua. 


\section{I fenomeni di cambiamento di lingua: l'intrecciarsi delle situazioni bilingui ed esolingui}

Tipi di alternanza

\section{Come risorsa comunicativa («di tipo esolingue») Come effetto espressivo («di tipo bilingues)}

\section{Alternanza (cambiamento di lingua momentaneo)}

Indica difficoltà nella competenza linguistica

Indica, per esempio, elementi dell' intrarepertorio ${ }^{3}$ condiviso

L'alternanza può svolgere due funzioni diverse: da un lato, può rappresentare una «marca» linguistica dell 'esistenza di una deficienza nella competenza linguistica (per cui corrisponderebbe a semplici risorse comunicative); dall'altro, può costituire una risorsa espressiva, vale a dire un'aggiunta di significato al significato già contenuto nella forma linguistica. Q uesta procedura è tipica delle situazioni bilingui. In ogni caso, gli esempi che si forniranno in seguito rappresentano evidenti intrecci tra il tipo di situazione e il tipo di alternanza sul modello: situazione esolingue/alternanza «di tipo esolingue», ma anche situazione esolingue/ alternanza «di tipo bilingue».

\section{L'alternanza}

\subsection{L'alternanza «di tipo bilingue» (come risorsa espressiva)}

(I) Brano di conversazione raccolto in N uova Zelanda, in situazione bilingue, da Kinder (1985), in Bettoni (1992):

[... ] non ho io conoscenze come le dicevo prima come uno scapolo (.) che esce di più che va ai parti e non parti e feste e non feste così no?

(II) Brano di conversazione raccolto presso la EOIBD ${ }^{4}$ in situazione esolingue, corpus Rêver en classe 5 (T orrens, 1994):

3. Secondo la sociolinguistica (Gumperz, 1968), il repertorio corrisponde a tutte le varietà del parlato, e l'intrarepertorio alla variazione linguistica individuale di ciascuno dei parlanti.

4. Escola O ficial d'I diomes Barcelona-D rassanes.

5. II corpus Rêver en classe è ottenuto tramite la registrazione di una lezione di italiano lingua straniera di primo corso intensivo presso I'EO IBD, in data 3-XII-1993 - 30 mese, che corrisponde al quinto mese di un corso normale. Le alunne, una decina, non erano a conoscenza del fatto che i loro dialoghi fossero registrati. La raccolta del corpus viene realizzata dall' autrice del presente articolo, che corrisponde alla docente (R.).

L'attività realizzata richiede una rilevante negoziazione del significato e del significante degli elementi lessici necessari alle alunne per la real izzazione dell'attività: le alunne devono identificare e descrivere determinate voci, presentate su nastro magnetico e inserite in una conversazione registrata in ambito familiare. In seguito, le al unne fanno una descrizione fisica delle donne alle quali corrisponde ciascuna delle voci. Per concludere, la docente mostra le fotografie che corrispondono alle voci della registrazione. 
\#voci\#

R. allora quello che farò adesso è far vedere le foto

\#sorpresa\#

A. *ah

AA. *oh

R. è questo lo scopo dell'esercizio (.) alla fine si fanno vedere (.) allora (.) sono due quasi uguali ma non sono uguali (.) dovete (.) ah vediamo se scoprite chi sono (.) ve lo passo o lo lascio qui a $\mathrm{M}$ arta (.) voi guardate qui

A3. ah vale

A5. [esta es ella esta es ella]

A3. [una es ésta] y la otra $(X X X)$ ?

A6. a ver (.) eh la nonna

R. questa?

A4. ésta

$R$. una è questa e l'altra è questa

\#confusione\#.

[Rêv. (25), Lin. 442-456]

II primo esempio viene incluso da Bettoni (1992: 434-435), tra i prestiti «inutili» che subentrano «con il cedimento dei confini personali tra le due culture e l'abitudine ad alternare le due lingue» e che «si sanno benissimo». Effettivamente, questo esempio rientra, seguendo la tipologia da noi proposta, nelle alternanze «di tipo bilingue».

Per quanto riguarda il secondo esempio, I'alternanza la nonna corrisponde, pur trattandosi di apprendenti di primo livello, all'uso di un termine avente finalità espressive nella lingua oggetto e cioè come farebbe un parlante bilingue. In altre parole, l'item oggetto dell'alternanza è diventato un elemento dell'intrarepertori ${ }^{6}$ (come nell'esempio precedente) dei due interlocutori (A6 e A4) e, per questo motivo, può essere usato quale risorsa espressiva alla maniera dei bilingui.

Confrontando i due esempi che abbiamo fornito e avendone individuati moltissimi altri che la mancanza di spazio non ci permette di presentare, possiamo concludere che i discenti, pur trovandosi in una situazione esolingue, dimostrano in certe occasioni un comportamento linguistico caratteristico delle situazioni bilingui.

Immaginiamo un asse i cui punti estremi corrispondono da un lato, all'unilinguismo, dall'altro, al bilinguismo (D i Pietro, 1988): su questo asse si colloca il discente, che progredisce dallo stadio di unilinguismo, nella propria lingua madre, allo stadio di bilinguismo. E non solo progredisce, ma si colloca anche volontariamente piuttosto verso un polo o piuttosto verso l'altro. ${ }^{7}$

6. V. nota 4.

7. In uno studio relativo ad emigrati latinoamericani a Parigi (banca dati della European Science Foundation), V asseur (1991) afferma (la traduzione dal francese è mia):

«G li elementi in L1 chelo straniero utilizza all'interno di una conversazione con un parlante nativo possono assumere il valore di interferenze se la conversazione si colloca sul polo esolingue, ossia, se i partecipanti la concepiscono come esolingue, oppure il val ore di «alternanze di codices se la conversazione tende piuttosto al polo bilingue. Q uesta variazione risulta evidente nel confrontare le diverse interazioni che lo stesso locutore ha intrapreso 


\subsection{Le alternanze «di tipo esolingue» (comerisorsa comunicativa)}

A questo punto vorremmo fare due precisazioni:

In primo luogo, alcuni alunni hanno probabilmente una predisposizione maggiore a sentirsi bilingui fin dai primi momenti. In secondo luogo, il significato dei cambiamenti di lingua e, in particolare, delle alternanze, varia nel tempo: sembra che nei primi livelli di apprendimento siano più comuni le alternanze «di tipo esolingue» e che man mano che il discente progredisce nell'apprendimento, diventino più comuni le alternanze «di tipo bilingue». M i occuperò, da questo momento in poi, esclusivamente di quei cambiamenti di lingua più legati alle deficienze nella competenza linguistica dei discenti, vale a dire delle alternanze di «tipo esolingue».

(III) Brano di conversazione raccolta presso l'U niversità di Potenza, Corpus Comentando (T orrens, 1994)8, in situazione esolingue:

Rm. sí «por *donde te podrás meter y salir lu*ciérnaga» luciérnaga? qué es una luciernaga?

\#sottovoce inintelligibile di C. e D.\#

$D$. euh un animale

$\mathrm{Rm}$. sí (.) es el animal ese que en la oscuridad (.) se enciende una luz

D. ah ah ah ah (.) lucciola

C. ah lucciola (.) ah.

[Com. (13) Lin. 368-375]

(IV) Brano di conversazione raccolta presso I'U niversità di Potenza (Corpus Comentando (Torrens, 1994), in situazione esolingue:

Rm. [... ] «Entregamos gratis nuestra energía, enriquecemos y estimulamos a los que $n^{\left({ }^{\circ}\right)}$ se nos acercan, con nuestra agudeza, chispas, brillantes»

$D$. chispas (.) qué significa?

Rm. sc:!: scin scintille?

D. scintille.

[Com. (11) Lin. 246-251]

nell'arco di diversi anni, vale a direin diversi stadi di sviluppo della sua competenza bilingue. [... ] II ricorso alla L1, variabile, che i diversi partecipanti ei diversi livelli di competenza permettono, dipendono altresi dallo stesso locutoreapprendente, nella misura in cui questi può concepire una situazione comunicativa come piuttosto esolingue o come piuttosto bilingue e utilizzare la propria L 1 di conseguenza».

8. II corpus Comentando è stato ottenuto attraverso la registrazione di una lezione di spagnolo lingua straniera del terzo anno - 30 mese - presso I'U niversità degli Studi di Potenza, in data 27-I-1993. Le due alunne (D. e C.) non sapevano di essere registrate. La raccolta del corpus, come nel caso di Rêver, è stata realizzata dall'autrice del presente articolo, che coincide con la docente ( $\mathrm{Rm}$.).

L'attività realizzata equival ealla semplice lettura di un testo eal commento dello stesso. II corpus, data la natura polemica del testo da commentare, presenta un grado spiccato di negoziazione tematica, legato tal volta a determinati cambiamenti di lingua. 
N ell'esempio (III), le alternanze indicano una deficienza nella competenza linguistica. D iciamo, in primo luogo, che l'insegnante focalizza la sua attenzione sul contenuto dell'item luciérnaga aprendo una sequenza metalinguistica. In seguito, D . utilizza l'alternanza come riformulazione intercodica ed evita cosi la riformulazione intracodica (nella L2, lo spagnolo). L'insegnante fornisce poi la riformulazione in L2 es el animal ese que en la oscuridad (.) se enciende una luz e D. ricorre di nuovo all'alternanza come riformulazione intercodica (lucciola).

$\mathrm{N}$ ell'esempio (IV) è invece l'insegnante a usare l'alternanza. La funzione di questo cambiamento di lingua risiede nell'evitare di fornire una riformulazione intracodica nella L2 (lo spagnolo) dell'item chispas, che risulterebbe particolarmente difficile.

Sia in questo frammento che nel precedente, la riformulazione equivale a una strategia di facilitazione, ${ }^{9}$ quale strategia di insegnamento/apprendimento, che sembra comunemente accettata da ambedue le parti.

L'esempio (V) rappresenta forse uno dei passaggi più interessanti per quanto riguarda le alternanze individuate nell'insieme dei due corpus che ho trascritto e studiato. Q uesto interesse risiede nella complessità dell'articolazione del significato, e nell'altissimo grado di negoziazione del significato e del significante che portano a termine, nella stessa misura, le due alunne e l'insegnante mediante riformulazioni intracodiche ed alternanze. Q uesto tipo di sequenze è di grande ricchezza dato l'altissimo grado di coinvolgimento dell'insegnante e delle alunne, dovuto all'uso ricorrente di strategie di intercomprensione.

$\mathrm{N}$ ell'esempio trascritto rileviamo in grassetto le alternanze, tralasciando altri cambiamenti di lingua (in corsivo), e tra determinati simboli ( item che corrispondono indistintamente alla riformulazione intercodica (le stesse alternanze) ed intracodica, senza distinguere tra $\angle 1 \quad 0<2$ (in senso stretto) e interferenza.

9. La denominazione strategia di facilitazione è stata coniata da Alber-Py (1986):

«La simplicité du discours alloglotte ne se fonde pas sur une comparaison avec des discours "absents", mais sur une proprieté intrinsèque de la conversation exolingue, à savoir l'existence de déplacements continuels le long d'un axesimple/complexe, dessiné par les interlocuteurs eux-mêmes. Cet axe regroupe en partie celui des ajustements réci proques: I'alloglotte peut complexifier son discours pour le rapprocher des normes utilisées par son partenaire, lequel peut, de son côté, simplifier son discours por le rendre accessible. N ous disons "peut", car la facilitation utilise souvent des procédés qui ne relèvent pas de la simplification: la reformulation par exemple constitueun procédé de facilitation dans la mesure où elle multiplie les informations [... ]. La facilitation prend deux formes: I'autofacilitation consiste à se faciliter à soi-même le travail de verbalisation ou, plus généralement, la participation à une conversation. Q uant à I'héterofacilitation, elle a pour fonction de faciliter ce travail à l'interlocuteur». 
(V) Brano di conversazione raccolta presso la EO IBD, in situazione esolingue, corpus Rêver en classe (Torrens, 1994):

\section{A3. R. sideas fijas? (cast.) ideas fijass}

R. \#sottovoce\# ideas fijas?

A3. R. ideas fijas 0 sea

$\mathrm{R}$. dimmelo in un altro modo (.) perché

A3. (o sea: che:) (.) sí (.) o sea no: variable (.) em:=

$\mathrm{R}$. =allora è come: come: $\mathrm{mm}$ : (.) testarudo (cast.) \& [U n po' X]

A3. [no] pero no quiero decir eso

R. no?

A3. no (.) (quiero decir) e: sicura sicura de ś misma

R. *ah (.) $\mathrm{m}$ :

A3. o sea con unasideas fijas [(que:)]

R. [sí sí (.) okay]

A3. mucho sicura (.) (que:)

\#T rascorrono approssimativamente trenta secondi. Sovrapposizione di voci inintelligibili\#

[... ]

R. $(X X X)$ in spagnolo

A3. (XXX) yo lellamo una persona de ideasfijas (.) o sea una persona segura (.) sicuras[perché:]

R. [sicura?] (.) sicura di se stessa.

A3. sí

R. questo può andare no? (.) sicura di sé [... ]

A11. no però (.) ma no è: questo $(X X X)$ segura di sé (.) pues segura de (XXX) de: de ideas fijass (è) che: no e: (o sea no e: che) (.) shai una=

R. =una idea

A11. una idea e: solo poi: fare questa idea (.) e idea fija è che: fa una: una idea de: una cosa che: vol (.) che (vole) fare e: ha de (.) ha de: fare questa cosa. R. ah

A11. che no: no puo cambia: per una cosa che: che dice:

R. allora una parafrasi perché io non (.) in italiano.

[Rêv. (22), Lin. 381-394 / 397-401 / 404-411]

La negoziazione prende spunto dalla richiesta di traduzione di un item, formulata da A3 nella L1 (ideas fijas, Lin. 381), che determina I'apertura di una sequenza metalinguistica. L'insegnante richiede in seguito ulteriori spiegazioni su tale item, e A3 le fornisce sotto forma di alternanza (no: variable, Lin. 385), affiancandosi a ideas fijas.

Per l'analisi dell'alternanza che segue occorre considerare in primo luogo che nelle seguenze metalinguistiche dei due corpus analizzati, allorquando la richiesta di traduzione viene formulata da un'alunna in modo esplicito, l'insegnante facilita regolarmente tale traduzione, vale a dire fornisce un equi- 
valente dello stesso item nella L2 (I'italiano), a meno che non si avvalga della cosiddetta strategia di aggiramento10 (Torrens, 1994). ${ }^{11}$

C iononostante, nell'esempio $\mathrm{V}$, anche l'insegnante ricorre all'alternanza testarudo (cast., Lin. 386), procedimento per nulla consono al suo modo di agire, per due precisi motivi: 1) in primo luogo è portata solitamente alla strategia di aggiramento; 2) in secondo luogo, osserva rigorosamente il principio: «[in aula di lingua straniera] si parli in L2». Comunque, data la difficoltà nell'articolazione del significato dell'item primigenio ideas fijas (Lin. 381), il cambiamento di lingua risulta lecito anche all'insegnante, che si sovrappone, nella complessa elaborazione del significato, ad altri modi, successivi, di riformulazione:

1. in L2 (sicura A3, Lin. 389/ mucho sicura A3, Lin. 393 /sicura di se stessa R., Lin. 399 /sicura di sé R., Lin. 401 /segura di sé A11, Lin. 404);

2. in L1 (con unasideasfijas A3, Lin. 391/ una persona de ideasfijas o sea una persona segura A3, Lin. 398/ segura de: de ideasfijas A3, Lin. 404);

3. con una lunga parafrasi intracodica (una idea e: solo poi : fare questa idea (.) eidea fija è che: fa una: una idea de: una cosa che: vol (.) che (vole) fare e: ha de (.) ha de: fare questa cosa [... ] che no: no puo cambia: per una cosa che: che dice: A11, Lin. 405-408).

Si noti, d'altro canto, che lo stesso insegnante richiede in modo esplicito, il cambiamento di lingua da parte delle alunne (XXX) in spagnolo (Lin. 397).

10. Strategia di aggiramento equivale, in spagnolo, a estrategia de evitación de proporción de ayuda (Torrens, 1994), e corrisponde a quella che utilizza la docente evitando di fornire all'alunno l'aiuto che ha precedentemente richiesto (solicitud de confirmación; solicitud de traducción).

11. Fornisce la traduzione, per esempio, nel brano che segue:

A11.che (.) che che si un uomo (.) un uomo o una donna (.) e penso che è molto (.)molto più gra

(.) agradable? agradable che la de do (.) della voce della donna (.) ela voce della donna me ricorda alla mia nonna (.) ma quando si como (.) como se dice (it.) enfadaba?

R. quando si arrabbiava

A11. quando si arrabbiava perché $\mathrm{mm}$ tenía una voce eh suave.

[Rêv. (17), Lin. 317-321]

In al tre occasioni, la docente risponde alla richiesta di traduzione mediante la cosiddetta strategia di aggiramento, con l'uso ricorrente di connettivi testuali:

A7 la donna pues $(X)$ mi ricorda la mia nonna e: (.) bueno tu has dicho que: \#ride\# que recordáramos (.) pue:s a: la mia nonna: e: è morta?

R. hm \#fa cenno di assentire\#

A3. è morta e: $\mathrm{m}$ mi ha ricordato de: cuando yo era: una: pic era era piccola e: la mia nonna: passava?

R. \#fa cenno di assentire\# H m

A7. restava: molti giorni: a: a casa mia=

$\mathrm{R}$. $=\mathrm{hm}$ \#fa cenno di assentire e chiede di continuare\#

A7. eallora: andiavamo? \#mormora e ride con altri\# andiavamo \#ride\# a la spiaggia che: è accanto: mi casa e: la mia casa e: bueno (.) giocava: con e (.) i (.) con un mio amici (..)

R. hm (.) bello no?

A7. sí (.) sí sí \#ride\#».

[Rêv. (11), Lin. 214-224] 


\section{Conclusioni}

Includo alcune conclusioni dagli esempi analizzati e dalle considerazioni di portata teorica che man mano ho evidenziato.

In primo luogo, la necessità di analizzare l'interazione discorsiva che intraprendono l'insegnante e gli alunni, la cui complessità risiede nell'interpretazione delle «marche» linguistiche che racchiude. Tra queste marche, i cambiamenti di lingua. L'alternanza, in particolare, pur essendo caratteristica nella conversazione esolingue, sia nel «tipo esolingue» che nel «tipo bilingue» compare in situazione, a sua volta, bilingue ed esolingue e si verificano interessanti intrecci (come alternanza «di tipo bilingue»/situazione esolingue) ${ }^{12}$. D i conseguenza, in certe occasioni l'alternanza sta ad indicare che l'alunno si colloca in situazione bilingue, e cosi deve essere inteso dall'insegnante; in altre occasioni invece equivale a concrete strategie di insegnamento/apprendimento che, una volta identificate, potrebbero essere gestite dall'insegnante in modo proficuo.

Infine, vorrei indicare che, come notato già da Vasseur (1991), I'affinità tra la L1 e la L2 sembra favorire il ricorso al cambiamento di lingua, ed essendo l'italiano e lo spagnolo o il catalano particolarmente simili, le nostre possibilità di ricerca aumentano considerevolmente.

\section{Annesso: convenzioni di trascrizione ${ }^{13}$}

a) Sovrapposizione parziale di turni

[testo]

b) Informazione extralinguistica (per esempio la gestualità)

\#lesto\#

c) Allungamento vocalico o consonantico

vocale:/ consonante:

d) T rascrizione ipotetica

parola probabile (testo)

quantità probabile di sillabe (XXX)

e) Enfasi nell'intonazione

*testo/ tes*to

f) Intonazione interrogativa

testo?

12. Comenell'esempio II. Per quanto riguarda altri tipi di cambiamento di lingua come la c.d. commutazione (N ussbaum, 1992), caratteristica invece nella conversazione bilingue, abbiamo verificato altresi i soprannominati intrecci.

13. Le convenzioni di trascrizione provengono, in parte, da O rletti -T esta (1991), che presentano un elenco particolareggiato, nell'appendice del loro articolo, dei simboli utilizzati da diversi autori. N oi abbiamo usato, in linea di massima, quelli proposti da G umperz (1982); G umperz-Berenz (1990), e quelli usati per la banca dati della European Science Foundation (1990). 


\section{g) Discesa nel tono \\ Ltesto \\ tes\to \\ h) Allacciamenti \\ testo $=$

$$
\text { testo }
$$

i) Pause
(.) fino a $1 \mathrm{sec}$.
(..) fino a 2 sec.
(... ) fino a $3 \mathrm{sec}$.

\section{Bibliografia}

AA.VV. (1991). 8eme Colloque international: acquisition des langues. Perspectives et recherches (16-18 maggio).

AA.VV. (1992). «Introduzione all'italiano contemporaneo». Vol. I. Bari: Laterza.

ALBER, J.L.; PY, B. (1986). «V ers un modèle exolingue de la comunication interculturelle». Etudes de Linguistique appliquée, 61: 78-90.

BetTonI, C. (1992). «taliano fuori d'Italia». In AA.VV. (1992): 411-460.

DI PIETRO, J.F. (1988). «V ers une tipologie des contacts linguistiques». Language et Societé, 43.

DILL, A.S. (1971). Language in Social Groups. Essays by J.J. Gumperz. Stanford U niversity Press

G Um PERZ, J.J. (1968). «T he Speech Comunity». In Dill: 114-118.

N ussBaum, L. (1992). «M anifestacions del contacte de llengües en la interlocució». Treballs de sociolingüística catalana, 10.

O RLETTI, F.; TESTA, R.(1991). «La trascrizione di un corpus di interlingua: aspetti teorici e metodologici». Studi italiani di linguistica teorica ed applicata, 2.

TO RRENS, R. (1994). Las estrategias de intercomprensión y de enseñanza/aprendizaje en el aula de italiano y español lengua extranjera: un estudio de los cambios de lengua. Treball de recerca (inedito). Universitat de Barcelona, Facultat de Filologia Romànica, Àrea de Lingüística.

- (Forthcoming). «The Italian Community in Spain: Integration and Language Interaction». In Turell, M .T. (ed.). M ultilingualism in Spain. Philadelphia: Clarendon.

- (Forthcoming). «Facilitating Procedures and their Interpretation by Speakers. The Role of $\mathrm{L} 1$ and its Relation with Language Awareness;. In Language Aware ness. Philadelphia: Clarendon.

VASSEUR, M .T . (1991). «Entre exolinguisme et bilinguisme, l'acquisition d'une langue étrangere». In AA.VV. 1991. 